\title{
Intermittent signs of erythromelalgia diagnosed by smartphone selfie
}

\begin{abstract}
Erythromelalgia, a rare condition associated with myeloproliferative disorders, causes intermittent ischemic pain in the extremities. A 42-year-old woman presented to our hospital with severe bilateral leg pain and intermittent red-violet skin discoloration for 4 years. A Smartphone "selfie" can accurately record the intermittent signs of erythromelalgia, enabling correct diagnosis of this uncommon condition.
\end{abstract}

Keywords: erythromelalgia, selfie, smartphone, essential thrombocytosis, myeloproliferative disorders, intermittent signs, polypharmacy, remote medicine, telemedicine, mhealth
Volume I Issue I - 2017

\author{
Hiroki Maita, 'Tadashi Kobayashi, ${ }^{2}$ Hiroshi \\ Osawa, ${ }^{2}$ Takahiro Hirano, ${ }^{3}$ Hiroyuki Kato ${ }^{3}$ \\ 'Development of Community Healthcare, Hirosaki University \\ Graduate School of Medicine, Hirosaki-shi, Japan \\ ${ }^{2}$ Department of General Medicine, Hirosaki University School \\ of Medicine \& Hospital, Hirosaki-shi, Japan \\ ${ }^{3}$ General Medicine, Hirosaki University Graduate School of \\ Medicine, Hirosaki-shi, Japan
}

Correspondence: Hiroki Maita, Development of Community Healthcare, Hirosaki University Graduate School of Medicine, Japan, Tel +8|-|72-33-5| |I, Fax +8|-|72-39-8|89, Email maita@hirosaki-u.ac.jp

Received: July 21, 2017 | Published: September 08, 2017

\section{Introduction}

Erythromelalgia is a rare condition associated with myeloproliferative disorders such as essential thrombocythemia or polycythemia vera. $^{1}$ Erythromelalgia causes microvascular thrombotic occlusions induced by platelet hyperaggregability in acral vessels, ${ }^{2}$ with intermittent ischemic symptoms presenting as a triad of redness, warmth, and pain of the extremities. ${ }^{3}$ Aspirin, a common and inexpensive drug is the first choice of therapy. ${ }^{1,2}$ It is difficult to record the typically intermittent skin findings noted in this condition. ${ }^{4}$ We describe how a photo taken by a patient on her smartphone (selfie) established the correct diagnosis, and helped reduce the use of multiple analgesics.

\section{Case presentation}

A 42-year-old woman presented to our hospital with intermittent severe bilateral leg pain and red-violet skin discoloration for 4 years. She was known to have essential thrombocytosis for 7 years. High doses of analgesics (loxoprofen 600mg/day, pregabalin 100mg/day) provided temporary relief. Physical examination in the outpatient department revealed normal skin findings and no ischemic signs in her legs. We instructed her to take pictures of her abnormal skin findings during an attack. Paroxysmal skin findings noted on a photograph she took using her smartphone, (Figure 1) revealed erythromelalgia. Oral aspirin $(100 \mathrm{mg} /$ day) relieved her pain and reduced the need for other analgesics.

\section{Discussion}

The patient's smartphone, a product commercially available in Japan without need for additional software, recorded her typically intermittent abnormal skin findings, established a diagnosis of erythromelalgia, and reduced polypharmacy with analgesics. Recording of definitive skin findings at the time of a patient visit is difficult because erythromelalgia typically shows intermittent symptoms. ${ }^{4,5}$ Once diagnosis is confirmed, aspirin is an inexpensive treatment option compared to expensive analgesics. A smartphone "selfie" can accurately record the intermittent signs of erythromelalgia, enabling correct diagnosis of this uncommon condition. We believe that "selfies" will become more useful in general practice, as well as in telemedicine, in the future.

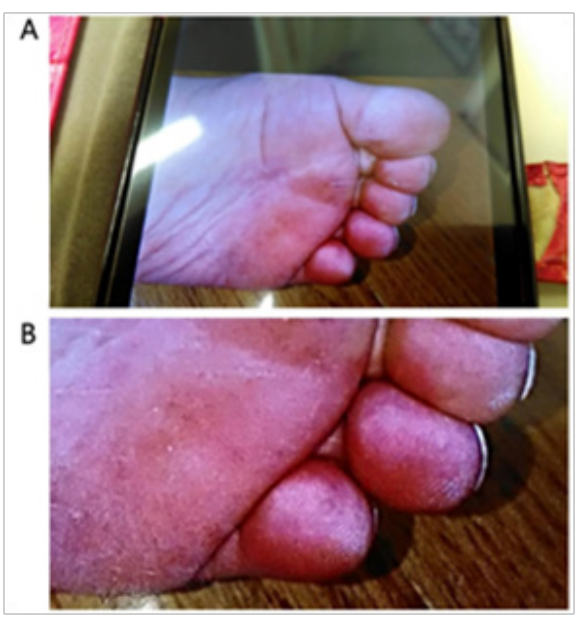

Figure I Photograph of Patient's Left Toe using her Smartphone Selfie. Edema and Erythema during attacks are found in the Third to Fifth Toes and Sole of the Foot.

a. Picture of Patient's Smartphone Screen Taken by a Physician in an Outpatient Department.

b. An Enlarged Image of Photo A.

\section{Patient consent form}

Written informed consent for patient information and images to be published was provided by the patient.

\section{Acknowledgements}

None. 


\section{Conflict of interest}

The authors declared no potential conflicts of interest with respect to the research, authorship, and/or publication of this article.

\section{References}

1. Kurzrock R, Cohen PR. Erythromelalgia and Myeloproliferative Disorders. Arch Intern Med. 1989;149(1):105-109.

2. Michiels JJ, Johannes A, Steketee J, et al. Erythromelalgia Caused by Platelet-mediated Arteriolar Inflammation and Thrombosis in Thrombocythemia. Ann Intern Med. 1985;102(4):466-471.
3. Davis MD, O Fallon WM, Rogers RS, et al. Natural History of Erythromelalgia: Presentation and Outcome in 168 Patients. Arch Dermatol. 2000;136(3):330-336.

4. Cohen JS. Erythromelalgia: New Theories and New Therapies. $J$ Am Acad Dermatol. 2000;43(5):841-847.

5. Norton JV, Zager E, Grady JF. Erythromelalgia: Diagnosis and Classification. Foot and Ankle Surg. 1999;38(3):238-241. 